\title{
Lectura crítica de artículos científicos basados en las temáticas de densidad y flotabilidad
}

Diego Armando Sanabria Yaruro *

Artículo de investigación

Fecha de Recepción: 6 agosto 2017.

Fecha de Aprobación: 15 enero 2018.

\section{Resumen}

Lafinalidadde esteartículodeinvestigación científica y tecnológica, es presentar los resultados de la investigación realizada en la Escuela Normal Superior Antonia Santos (ENSAS); donde las pruebas SABER 11 del año 2015, arrojaron como resultado, que el $76 \%$ de los estudiantes obtuvieron 60 puntos o menos en lectura crítica. Para tratar de dar solución a la necesidad planteada, se buscó identificar el impacto de la aplicación de talleres, en la lectura crítica de artículos científicos, basados en las temáticas de densidad y flotabilidad en $7^{\circ}$.

La investigación es del tipo investigación acción participativa con enfoque mixto, de tipo experimental. Se realiza un pretest como diagnóstico; luego, la intervención con la aplicación de tres talleres de lectura crítica; finalmente, se procede a analizar la evolución en comprensión lectora de los estudiantes.

Los estudiantes vistos de manera grupal, alcanzaron un aumento del $8,7 \%$ en el nivel de lectura literal; del $1,5 \%$, en el nivel inferencial; y del $1 \%$, en el nivel crítico. Por lo que, la ejecución de talleres enfocados en lectura crítica, direccionados desde el constructivismo y la pedagogía crítica, pueden ser un camino para fortalecer la competencia de lectura crítica de artículos científicos, en los estudiantes.

Palabras clave: pedagogía crítica, constructivismo, lectura crítica, artículo científico, densidad, flotabilidad.
* Escuela Normal Superior Antonia Santos - Santander - Colombia 


\section{Introducción}

Desde finales del siglo pasado e inicios del presente siglo, en diferentes países del mundo, entre ellos Colombia, se han iniciado una serie de reformas educativas con el propósito de dar un giro en la educación, buscando pasar de un modelo de enseñanza de conocimientos (transferencia de contenidos) a un modelo donde se pretende desarrollar en el estudiante competencias del tipolaboral, ambiental y ciudadanas, como lo promueven instituciones del orden internacional, la Organización para la Cooperación y el Desarrollo Económico (OCDE) y el Fondo Monetario Internacional (FMI).

Aunque este enfoque se corresponde con la idea de mercantilización del ser humano y la alienación de la cultura, se debe tener en cuenta, que el proceso de globalización que se vive en la actualidad, y siendo una realidad que cobija a la humanidad, implica que los estudiantes se encuentran en un mundo "competitivo", donde cada uno muestra lo mejor de sí, para alcanzar el lugar que desea en la sociedad. De esta manera, y con el fin de que el estudiante en formación tome consciencia de la realidad que le rodea, sepa interpretarla, se haga consciente y logre intervenirla desde sus decisiones para actuar; la lectura crítica planteada desde el modelo de competencias, es una habilidad que puede ayudar a que el estudiante lleve a cabo la liberación de la consciencia, como lo promovería Paulo Freire en la pedagogía de la liberación o la pedagogía emancipadora.
Teniendo en cuenta lo anterior, el gobierno colombiano ha decidido encaminarse a la integración de la OCDE, y para ello participa de las pruebas PISA, organizadas por dicha entidad internacional; donde se mide el grado de competencias alcanzado por los estudiantes de los países participantes en la prueba, en edad de quince años. Dichas pruebas se aplican cada tres años, iniciando en el año 2000 con énfasis en lectura, la siguiente prueba tuvo por énfasis las Ciencias Naturales, y la siguiente fue en Matemáticas; este ciclo se repite desde entonces continuamente.

Según análisis realizados por Julián de Zubiría, a los resultados de las prueba PISA en el año 2015, en Colombia solo tres de cada mil jóvenes escolarizados tienen un nivel de lectura crítica a los 15 años. Desde esta perspectiva, y analizando los resultados obtenidos en la institución educativa objeto del presente estudio, en las pruebas saber 11 para el año 2015, se encuentra que para el caso de lectura crítica, solo 28 estudiantes de 117 que presentaron la prueba saber 11, obtuvieron una calificación que oscila entre los 60 y 85 puntos, lo que sugiere la necesidad de abordar la situación, buscando estrategias que promuevan y fortalezcan el desarrollo de la competencia en lectura crítica. Estos resultados concuerdan con los presentados por estudiantes colombianos en PISA, si se tiene en cuenta que las pruebas SABER son presentadas por estudiantes que oscilan en su mayoría, en edades comprendidas entre los 16 y 18 años, por lo que el desarrollo de dicha 
competencia se debe enfocar desde edades mucho más tempranas que los 15 años.

Debido al problema planteado, surge la necesidad de generar respuesta a la pregunta ¿Cómo impacta la aplicación de talleres de lectura crítica de artículos científicos, basados en las temáticas de densidad y flotabilidad, en los niveles de lectura en estudiantes de $7^{\circ}$ de la Escuela Normal Superior Antonia Santos?, lo que llevó a plantear como objetivo general, identificar el impacto de la aplicación de talleres en lectura crítica de artículos científicos, basados en las temáticas de densidad y flotabilidad, en los niveles de lectura en estudiantes de $7^{\circ}$ de la ENSAS. Para alcanzar esto, se elaboraron tres objetivos específicos, primero, identificar la capacidad de lectura crítica de los estudiantes de $7^{\circ}$ de la Escuela Normal Superior Antonia Santos, en artículos científicos basados en las temáticas de densidad y flotabilidad; en segundo lugar, diseñar y aplicar una estrategia de lectura crítica de artículos científicos basados en las temáticas de densidad y flotabilidad en $7^{\circ}$; y el tercer objetivo, analizar la evolución en el nivel de lectura crítica de artículos científicos basados en las temáticas de densidad y flotabilidad en $7^{\circ}$ una vez implementada la estrategia enfocada en este tipo de lectura.

Siguiendo el sentido del pensamiento Freiriano, en cuanto a que el estudiante se debe convertir en el eje central del proceso lector (Freire, 1989), y de igual manera, las ideas del constructivismo sociohistórico cultural en cuanto a la construcción del conocimiento, el taller ejecutado en mesa redonda, posibilita la interacción multidireccional entre estudiantes y docente, donde el estudiante se acoge como el actor principal o centro de la actividad pedagógica, al tiempo que de la interacción sociocultural de todos los participantes en el aula, se erigen los cimientos en la construcción de ese nuevo conocimiento, es decir, desde sus presaberes compartidos, cada estudiante va consolidando los nuevos conceptos de densidad y flotabilidad.

Por otro lado, los estudios relacionados con la dificultad que entraña la enseñanza-aprendizaje del concepto de flotabilidad y densidad, han sido diversos en los últimos años, siendo tema de investigación desde hace mucho tiempo; entre ellos, se pueden mencionar los realizados por Barral (1990), García (1998), Mazzitelli, Maturano, Núñez \& Pereira (2006), Madrigal \& Slisko (2010) y Aguilar (2011), que unido a los resultados poco satisfactorios de los estudiantes en el área de las ciencias naturales, tanto en el aula como en las pruebas SABER obtenidos en la ENSAS para el año 2015, motivan a realizar esta investigación donde se integra la lectura crítica con artículos científicos que abordan los temas de densidad y flotabilidad, sin desconocer que la lectura crítica es una competencia genérica y, por tanto, la base del aprendizaje significativo de las diferentes áreas del conocimiento.

En cuanto a los antecedentes, existen diversas investigaciones con enfoque en el fomento de la lectura crítica, entre ellas se destaca "La secuencia didáctica
Debido al problema planteado, surge la necesidad de generar respuesta a la pregunta ¿Cómo impacta la aplicación de talleres de lectura crítica de artículos científicos, basados en las temáticas de densidad y flotabilidad, en los niveles de lectura en estudiantes de $7^{\circ}$ de la Escuela Normal Superior Antonia Santos? 
como estrategia para desarrollar niveles de lectura crítica en estudiantes de grado quinto" desarrollada en el año 2012 en la Universidad Minuto de Dios, ejecutada por Arias, Beltrán \& Solano, la misma tuvo como objetivo, potenciar la lectura crítica en los estudiantes del grado Quinto de Primaria del Colegio la Inmaculada de Chía mediante la aplicación de la secuencia didáctica como estrategia, encontrando que la habilidad de lectura crítica tuvo un incremento del 11,9 \% una vez aplicada la estrategia, en comparación con los datos recolectados en el diagnóstico, este y otros antecedentes serán abordados en el marco teórico.

En lo referido a densidad y flotabilidad y sus antecedentes, Barral (1990) en la Universidad Santiago de Compostela, desarrolló un trabajo investigativo para determinar la concepción de los estudiantes sobre, ¿cómo flotan en el agua los cuerpos que flotan?; en los niveles primaria, secundaria y universitario, del cual concluyó que se debe dar importancia a las ideas de los alumnos en torno al concepto de flotación y su resistencia al cambio de conceptualización y para que la enseñanza de las ciencias mejore, del alumno se requiere mayor esfuerzo de reflexión y comunicación de sus ideas, entre otros aspectos, y del profesor a interesarse en las ideas "no aceptables" de los estudiantes y mayor compromiso en el tiempo dedicado a un determinado tema, discusiones en pequeño y gran grupo, así como el trabajo continuo sobre la revisión del currículo de acuerdo con el avance logrado y el cambio en la evaluación.
En cuanto a los referentes teóricos, se ha tomado a Lev Vigotsky desde la teoría sociohistórico - cultural, con el enfoque pedagógico constructivista, el cual se posibilita como un camino hacia la formación de estudiantes autónomos, competitivos, con capacidad crítica y de intervención en la realidad en la que se desenvuelve, la sociedad se encuentra en constante cambio y por ello necesita seres humanos con capacidades autónomas para reinventarse $\mathrm{y}$ amoldarse a las nuevas condiciones. En cuanto a la pedagogía, el enfoque está direccionado por la pedagogía crítica de Freire, donde el docente desempeña el papel orientador en la formación de los estudiantes y los acompaña en el proceso de liberación y emancipación mediante el proceso dialógico, donde cada actor educativo participa y aporta en la construcción de su formación. Estos dos referentes teóricos pueden ser articulados mediante el desarrollo de las habilidades de lectura crítica orientada desde el pensamiento de Daniel Cassany, donde el estudiante con sus conocimientos previos, determinados por la sociedad y la cultura en la que se ha desenvuelto, y la integración con sus compañeros y el docente, recorre el camino hacia la develación de la verdad y las intenciones del autor.

En lo relacionado con la metodología, esta investigación se enmarca a la línea de investigación lectura y escritura en educación, del tipo Investigación Acción Educativa, con diseño experimental y enfoque mixto. Para la realización del diagnóstico, se empleó una prueba tipo SABER, denominada, pretest, con la que se identificaron los niveles de 
lectura, en el caso de la intervención se ejecutaron tres talleres, en los cuales los estudiantes pudieron dar respuesta a preguntas abiertas, basadas en lecturas de artículos científicos orientados desde las temáticas de densidad y flotabilidad; para finalizar y evaluar el grado de desarrollo de la habilidad crítica alcanzado a través de los talleres, se aplicó el postest, el cual es idéntico al pretest.

Para observar cómo puede influir la aplicación de la misma prueba como pretesty postest, las mismas, en idénticos tiempos, se aplicaron en un grupo de control. Para el análisis de datos en el caso de los tests, estos se realizaron de acuerdo con el número de aciertos y desaciertos para el cuestionario; y en el caso de los talleres de intervención, los mismos se calificaron cualitativamente de acuerdo con la escala nacional y su equivalencia con la escala institucional.

La lectura crítica es una competencia, necesaria en los estudiantes para transitar en el camino hacia la liberación de la consciencia, hacia la toma de consciencia de la realidad, desde el desenmascaramiento del autor y sus ideas, sus pretensiones e intenciones, una habilidad necesaria hoy día e indispensable, si se tiene en cuenta el mundo globalizado en el que se vive y el flujo de información a través de los diferentes discursos por diferentes medios de comunicación, esto nos lleva entonces, a que los estudiantes deben poder acceder a nuevas y más profundas maneras de hacer lectura del discurso, lo que les dotará de la capacidad para discernir las ideas y tomar posiciones y decisiones en torno a ellas, afectando de esta manera la realidad que los acoge.

\section{Marco teórico}

\section{Formación en competencias, constructivismo, pedagogía crítica y lectura crítica}

La educación por competencias se ha propuesto como una forma para que el estudiante se convierta en capital cultural, es decir, tenga desarrollo personal; capital social, que incluye la participación ciudadana, y en capital humano o capacidad para ser productivo (Dirección General de Educación y Cultura de la Comisión Europea, 2004), y según esta visión, el ser humano se formará bajo unas competencias estandarizadas con fines estrictamente económicos, por lo que de esta manera, este sistema educativo se empeña en homogenizar y estandarizar al individuo; afectando la diferencia en la identidad cultural de la que hace parte cada persona, y la promoción de un modelo económico encaminado al mercantilismo, direccionando entonces los proyectos de vida, las ideas de esas personas, el libre desarrollo de su personalidad, de alguna manera afectando su ser (gustos, intenciones, forma de ver la vida y propósitos) y los encaja en parámetros idílicos de un modelo económico. Por lo tanto, se da un proceso de deshumanización y pérdida de identidad, cerrándose a un proceso estrictamente de homogenización de la forma de pensar y hacer la vida, así como de interpretar la felicidad por que el objetivo es 
producir capital humano que genere industrialización,

El desarrollo tiene dos aspectos. Por una parte es un mito global en el que las sociedades que llegan a industrializarse alcanzan el bienestar, reducen sus desigualdades extremas y facilitan a los individuos el máximo de felicidad que puede dispensar una sociedad. Por otra parte, una concepción reduccionista, en la que el crecimiento económico es el motor necesario y suficiente de todos los desarrollos sociales, psíquicos y morales. Esa concepción tecnoeconómica ignora los problemas humanos de la identidad, de la comunidad, de la solidaridad de la cultura (Morín, Ciurana, \& Motta, 2006, p. 103).

Teniendo en cuenta, entonces, que la educación por competencias deshumaniza y aliena de alguna manera al estudiante deslegitimando su identidad cultural, una posibilidad que podría ayudar a mitigar esta situación es la de articular dicho modelo con el constructivismo, ya que este permite que el estudiante, teniendo en cuenta los conocimientos y habilidades aprendidas en comunidad, pueda desarrollar nuevos conocimientos, habilidades y también competencias, sin olvidar la cultura que ha permitido su formación como persona y lo ha caracterizado.

De esta manera, con respecto al análisis de las estrategias de enseñanza basadas en un enfoque constructivista, Martínez \& Zea (2004) dicen que:

Si la sociedad está en cambio permanente por efecto de la trans- formación del conocimiento se requiere para ello, la necesidad de transformar los modelos y esquemas de pensamiento, al respecto Lanz (1999), señala que, para lograrlo se necesita formar un ciudadano con un conjunto de competencias básicas, entre las cuales cabe mencionar: capacidad de opinar, participación activa y cooperativa, capacidad para criticar y crear, y la de producir intelectualmente, entre otras. En este sentido, para que se logren estas competencias, se requiere la participación del docente, que con una función integradora y el uso de herramientas de comunicación podrá lograr en sus alumnos la organización, la socialización del ser y el compromiso con el otro de manera constructiva (p. 70).

Desde la perspectiva constructivista:

La teoría sociocultural de Lev Vigotsky indica que el desarrollo del ser humano está íntimamente ligado con su interacción en el contexto sociohistórico-cultural... y como bien lo señala Moll (1993), para Vigotsky la educación implica el desarrollo potencial del sujeto, y la expresión y el crecimiento de la cultura humana (Chaves, 2001, p. 59).

Chaves (2001) analizando las implicaciones educativas de la teoría sociocultural de Vygotsky, encuentra que:

Los aportes teóricos de Lev Vigotsky son propuestas pertinentes para repensar la educación y la práctica pedagógica. Estos postulados coinciden en la importancia de respetar al ser humano en su diversidad 
cultural y de ofrecer actividades significativas para promover el desarrollo individual y colectivo con el propósito de formar personas críticas y creativas que propicien las transformaciones que requiere nuestra sociedad (p. 63).

Sin perder de vista que la formación de competencias está ligado a un modelo económico tendiente a la deshumanización y la pérdida de identidad cultural, es conveniente que el desarrollo de competencias en los estudiantes esté direccionado por un ambiente educativo que los humanice, por lo que la pedagogía crítica puede ser el camino que lleve en esa dirección. En cuanto a la humanización que promueve Freire en el ámbito educativo, Araujo, Betancourt, Gómez, González \& Pareja (2015) se refieren de la siguiente manera:

Freire habla de educar en la diversidad que consiste en orientar una pedagogía crítica y dialogada, humanista cargada de esperanza porque es el camino a la emancipación de los sujetos. El logro final sería la posibilidad de liberar la conciencia del sujeto de cualquier forma de sujeción. Freire propuso en su momento, al igual que Amarthya Sen en la actualidad que en la educación está la libertad real del sujeto y es de ahí donde se debe partir para conseguir su emancipación, con el fin que logre contribuir asertivamente en transformar la realidad política y social de su entorno (pp. 30-31).

No se debe olvidar que, para Freire, estar alfabetizado es pensar por sí mismo, y esto resulta peligroso, porque pensar significa también cuestionar las estructuras dominantes del poder (Alvarado, 2007, p. 10).

Paulo Freire en más de una ocasión se refiere a la experiencia de la lectura en el contexto educativo, inclusive la alfabetización y el acto de leer es el tema central en sus libros: Alfabetización. Lectura de la palabra y lectura de la realidad (Freire \& Macedo, 1989) y La importancia de leer en el proceso de liberación (1991). Freire en su obra analiza con detalle el proceso de la lectura y el trasfondo pedagógico y político de ésta.

Algunas afirmaciones de Freire respecto al sentido de la lectura, son:

- "el acto de estudiar no se reduce a la relación lector-libro, o lector-texto" (Freire, 1991, p. 51).

- "Es imposible leer texto alguno sin poseer una comprensión crítica del contexto al cual se refiere" (Freire \& Macedo, 1989, p. 137).

- "La comprensión del texto a ser alcanzada por su lectura crítica implica la percepción de relaciones entre el texto y el contexto" (Freire, 1991, p. 94).

- "La lectura del mundo precede siempre la lectura de la palabra y la lectura de ésta implica la continuidad de la lectura de aquél” (Freire, 1991, p. 105). (Alvarado, 2007, p. 9)

"Aunque sus orígenes filosóficos sean pretéritos y variados (Marx, Freire) $\mathrm{y}$ podamos encontrar referencias
Freire habla de educar en la diversidad que consiste en orientar una pedagogía crítica y dialogada, humanista cargada de esperanza porque es el camino a la emancipación de los sujetos. 
bibliográficas anteriores, la perspectiva crítica en la enseñanza/aprendizaje de la lectura, la escritura o el pensamiento se ha diseminado y generalizado en los últimos años.” (Cassany, 2003, p. 16).

Refiriéndose a la lectura crítica, Cassany (2003) señala:

La constatación de que existen varios grados o planos de lectura en un mismo texto es muy aceptada. Gray (1960; citado por Alderson, 2000) ya distingue intuitivamente entre leer 'las líneas', leer 'entre líneas' y leer 'detrás de las líneas', que Alderson refiere respectivamente a comprender el significado literal del texto, sus inferencias y hacer una evaluación crítica del mismo.

Leer las líneas se refiere estrictamente a la comprensión literal de las palabras que componen el fragmento, a la capacidad de decodificar su significado semántico: elegir la acepción adecuada al contexto, de entre las que incluye el diccionario, y obtener todos los semas pertinentes.

En un grado más complejo, leer entre líneas se refiere a la capacidad de recuperar los implícitos convocados en el texto, que contribuyen de manera decisiva a elaborar su coherencia global y a construir el significado relevante del escrito.

Finalmente, leer detrás de las líneas se refiere a la capacidad de comprender qué pretende el autor, por qué lo escribió, con qué otros discursos se relaciona (contexto, comunidad, etc.); y a poder articular una opinión personal res- pecto a las ideas que expone, con argumentos coincidentes o no. Se trata, sin duda, de una respuesta externa al texto, de un grado de comprensión que exige disponer de mucha más información de la que aporta el texto o de la que este reclama que el lector aporte (pp. 113-116).

Siguiendo las ideas de Cassany (2003), sobre la lectura crítica, se puede entender que:

- es un tipo lectura compleja o lectura profunda - que exige niveles más altos de comprensión;

- requiere los planos previos de comprensión (literal, inferencias, intenciones, etc.) del texto, $y$

- exige una suerte de respuesta personal externa del lector frente al texto (frente a su contenido, intención, punto de vista, etc.), donde se pone en juego entonces el contexto del lector, el texto y el autor (p. 17).

Para Serrano (2008), la lectura crítica:

Supone comprender diversos modos de interpretación, es decir, considerar los diversos significados que el texto esconde. Supone, en consecuencia, no aceptar a priori las ideas y razonamientos del autor, sin antes discutirlos reflexivamente, prestar atención cuidadosa a las diversas connotaciones de las palabras o de los enunciados, discrepar de cualquier afirmación, principio o teoría; combatir y cuestionar imprecisiones u opiniones contrarias; derivar implicaciones y presupuestos; 
identificar puntos de vista e intenciones y distinguir posiciones ( $\mathrm{p}$. 508).

Siendo así, la lectura crítica pretende cuestionar hasta qué grado son ciertas las afirmaciones y razonamientos planteados por el autor y la forma cómo esas ideas están permeando o pretenden influir sobre el pensamiento del lector mismo, de tal manera que el lector debe recurrir a las fuentes originarias del pensamiento, en llegado caso de ser necesario, para validar la información que tiene a mano $y$, de esta manera, tener certeza real de lo que se le pretende transmitir y la intencionalidad con que se hace.

\section{Marco metodológico}

\section{Diseño}

Esta investigación se enmarca en la línea de investigación lectura y escritura en educación, del tipo, Investigación Acción, con diseño experimental y enfoque mixto. La investigación acción, según Murillo (2010, pp. 3-4), se refiere a la gama de estrategias que se realizan para mejorar el sistema educativo, destacando a Elliott como el principal representante de la investigación acción en un enfoque interpretativo. Elliott (1993), define la investigación acción como una reflexión sobre las acciones humanas y las situaciones sociales vividas por el profesorado que tiene como objeto ampliar la comprensión (diagnóstico), de los docentes de sus problemas prácticos, es decir, que las acciones van encaminadas a modificar la situación inicial con el objeto de lograr una comprensión profunda de los problemas.

\section{Participantes}

\section{Delimitación de la investigación (población y muestra)}

El estudio se desarrolló en la Escuela Normal Superior Antonia Santos del municipio de Puente Nacional; (Santander, Colombia); en la metodología investigación acción participativa. Se delimitó la investigación tomando como referencia y objeto de estudio, el curso $7^{\circ} \mathrm{C}$ con las siguientes características: 11 hombres, 21 mujeres, en edades comprendidas entre los 11 años a 16 años, de estrato social uno al tres, según la clasificación generada por el SISBÉN.

En pro de desarrollar el trabajo de forma más comprensiva, los análisis y resultados se manejaron conforme a la siguiente clasificación:

1 Los estudiantes del curso $7^{\circ} \mathrm{C}$ de la institución se denominaron como estudiante $(\mathrm{E})$ - número $\left(\mathrm{N}^{\circ}\right)$, denotados con la sigla $\mathrm{EN}^{\circ}$, para el caso del estudiante 1 , este quedó codificado con la sigla E1.

2. Se desarrolló una clasificación sumada dependiendo el curso al que pertenece el estudiante. Para los fines de esta investigación, la muestra estuvo constituida por un solo curso, Séptimo C, el cual se denotó con la sigla SC.

Con fundamento en las siglas especificadas con anterioridad, cada 
estudiante quedó codificado de la siguiente manera; (ver tabla 1):

Tabla 1.

Codificación de estudiantes.

\begin{tabular}{|c|c|c|}
\hline Curso & N Estudiante & Código \\
\hline SC & E01 & SC01 \\
\hline
\end{tabular}

Fuente: elaboración propia.

De esta manera, el análisis de los datos se pudo hacer por comparación entre los estudiantes, separando los datos de cada uno de los estudiantes.

En esta serie de ideas, la presente investigación contó con una codificación específica para cada estudiante, la cual permitió manejar la información recolectada y analizada, con cierto grado de confidencialidad y privacidad, disminuyendo las posibles incidencias que pudieran afectar a los participantes del presente estudio. Promoviendo con esto, el respeto por el desarrollo cognitivo y actitudinal del ser humano, la libertad en su desarrollo y la igualdad en el trato. Es de anotar que, cada uno de los participantes contó con el consentimiento dado por el acudiente respectivo.

\section{Instrumentos}

La ejecución del proyecto de investigación bajo la metodología de la investigación acción, inicia aplicando una entrevista escrita a los docentes, en la cual se buscó conocer la manera cómo los docentes del área de ciencias naturales y lengua castellana de la ENSAS abordan la lectura de las ciencias naturales. De igual manera, se hizo un test autodiagnóstico a cada uno de los estudiantes, cuyo propósito fue conocer aspectos puntuales, acerca de la forma como los estudiantes realizan el proceso lector. Para finalizar la primera fase del proyecto referida al diagnóstico, se aplicó un examen tipo SABER a los estudiantes, el cual se denominó (para propósitos de esta investigación) Pretest; concluida esta primera parte y elaborado el diagnóstico, se procedió a realizar la segunda fase del proyecto, que consistió en hacer la intervención basada en tres talleres de lectura enfocados a trabajar la lectura crítica; y como tercera y última fase, se aplicó el Postest (que es el mismo examen inicial o Pretest), con el fin de observar la evolución de los estudiantes en cuanto al nivel lector.

El pretest, los tres talleres de intervención $\mathrm{y}$ el postest, se fundamentan en las orientaciones dadas por Daniel Cassany y colaboradoras, en torno a cómo desarrollar un adecuado fomento de la lectura, la cual describe en el libro enseñar lengua (1998), teoría que fundamenta dichos instrumentos, $\mathrm{y}$ donde el autor es claro al recomendar que toda lectura debe ir acompañada de preguntas, y que en el mejor de los casos, las mismas deben conocerse, antes de abordar la lectura del texto, con el fin de definir un rumbo sobre el cual navegar el texto; de igual manera, los tres talleres para el fortalecimiento de la lectura crítica, son tomados y adaptados en su estructura de la investigación, denominada "La Secuencia Didáctica Como Estrategia Para Desarrollar Niveles de Lectura Crítica en Estudiantes de Grado Quinto", realizada por Arias 
y colaboradores en el 2012, en la UNIMINUTO.

\section{Procedimiento o plan de análisis}

El pretest y postest (corresponden a la misma prueba, aplicada al inicio del proyecto, específicamente durante el diagnóstico, y al finalizar el mismo), se analizaron teniendo en cuenta que tan solo una de las cuatro respuestas es la acertada, para cada pregunta, y de acuerdo con esto, se realizó el cálculo de la puntuación obtenida, por cada estudiante, para cada nivel de lectura (literal, inferencial y crítico).
Así mismo, para cada uno de los tres talleres, con claro enfoque crítico, abordando 7 subcategorías (tabla 2), las mismas se analizaron desde la valoración cualitativa, una a una, teniendo en cuenta la escala de valoración institucional, que se muestra en la tabla 3 , con el fin de observar en principio cómo fue la evolución de cada estudiante, durante la ejecución de los talleres de intervención, y si estos resultados concuerdan con los puntajes arrojados en el postest. De esta manera, cada subcategoría recibió una calificación cualitativa de acuerdo con la escala nacional: Bajo (B), Básico (Bs), Alto (A) y Superior (S), según correspondiera.

Tabla 2.

Listado de subcategorías.

\begin{tabular}{|c|c|c|}
\hline $\mathbf{N}$ & Subcategorías & Código \\
\hline 1 & Valorar procedencia de los textos & VPT \\
\hline 2 & Contextualizar los textos & CT \\
\hline 3 & Diferenciar entre hechos y opiniones & DHO \\
\hline 4 & Considerar la posición del autor & CPA \\
\hline 5 & Establecer relaciones intertextuales & ERI \\
\hline 6 & Reconocer analogías & RA \\
\hline 7 & Asumir postura crítica & APC \\
\hline
\end{tabular}

Fuente: elaboración propia.

Tabla 3.

Equivalencias de la escala institucional con respecto a la escala nacional

\begin{tabular}{|c|c|c|}
\hline \multicolumn{3}{|c|}{ ESCALA DE VALORACIÓN MIXTA } \\
\hline ESCALA NACIONAL & $\begin{array}{c}\text { ESCALA } \\
\text { CUANTITATIVA }\end{array}$ & ESCALA CUALITATIVA \\
\hline Desempeño Superior & $90.0-100.0$ & \multirow{3}{*}{$\mathrm{A}=$ APROBÓ } \\
\hline Desempeño Alto & $80.0-89.99$ & \\
\hline Desempeño Básico & $60.0-79.99$ & \\
\hline Desempeño Bajo & $00.0-59.99$ & NA = NO APROBÓ \\
\hline
\end{tabular}

Fuente: PEI de la ENSAS, 2009 
La denominación desempeño básico se entiende como la superación de los desempeños necesarios en relación con las áreas obligatorias y fundamentales, teniendo como referente los estándares básicos, las orientaciones y lineamientos expedidos por el Ministerio de Educación Nacional y lo establecido en el proyecto educativo institucional. El desempeño bajo se entiende como la no superación de los mismos (Decreto 1290, 2009, p. 2).

\section{Resultados}

\section{Comportamiento del grupo control sobre la prueba SABER (Pretest-Postest)}

Conocer el estado inicial del nivel de lectura de los estudiantes (Diagnóstico), en el caso de las tres dimensiones lectoras (literal, inferencial y crítico), es un punto de partida que contribuye, en la observación del proceso de intervención, al permitir identificar la tendencia de la evolución en los estudiantes por comparación entre el pretest y el postest.

Los resultados arrojados por el grupo control, durante la segunda aplicación del pretest denominado en esta fase postest, tabla 5 , arroja resultados muy similares a la primera aplicación de diagnóstico (pretest), tabla 4, los cuales no alcanzan a variar en más del $1 \%$, tanto para el nivel literal $(59,11 \%$ vs. $58,16 \%$ ), como para el inferencial $(38,42 \%$ vs. $38,27 \%)$; ya para el nivel crítico, la variación fue del 2,96 $\%$, pasando inicialmente de tener un porcentajes de aciertos a nivel grupal del $31,53 \%$ a $28,57 \%$, lo que sugiere que el conocer con anterioridad la prueba, desarrollándola bajo las mismas condiciones de aplicación, los resultados no varían significativamente en el posible incremento del número de aciertos a nivel grupal, por lo que no se considera realizar correcciones sobre los resultados obtenidos para el postest del grupo de investigación; y, por tanto, se hace una comparación en igualdad de condiciones entre el pretest y el postest. Es de resaltar que, el promedio de aciertos grupal disminuyó en décimas para el nivel literal e inferencial, y en enteros (el $2,96 \%)$ para el nivel de lectura crítica, en la aplicación del postest para el grupo control.

Tabla 4.

Promedio grupal de aciertos y porcentajes por nivel de lectura, pretest-grupo control.

\begin{tabular}{|l|c|c|c|c|c|c|}
\hline & \multicolumn{7}{|c|}{ Aciertos por nivel de lectura } \\
& Literal & $\begin{array}{l}\text { Porcentaje } \\
\mathbf{( \% )}\end{array}$ & Inferencial & $\begin{array}{c}\text { Porcentaje } \\
(\%)\end{array}$ & Crítica & $\begin{array}{c}\text { Porcentaje } \\
(\%)\end{array}$ \\
\hline Promedio Grupal & 4,14 & 59,11 & 2,69 & 38,42 & 2,21 & 31,53 \\
\hline
\end{tabular}

Fuente: elaboración propia. 
Tabla 5.

Promedio grupal de aciertos y porcentajes por nivel de lectura-Postest del grupo control.

\begin{tabular}{|l|c|c|c|c|c|c|}
\hline & \multicolumn{6}{|c|}{ Aciertos por nivel de lectura } \\
\cline { 2 - 7 } & Literal & $\begin{array}{l}\text { Porcentaje } \\
(\%)\end{array}$ & Inferencial & $\begin{array}{l}\text { Porcentaje } \\
(\%)\end{array}$ & Crítica & Porcentaje (\%) \\
\hline Promedio Grupal & 4,07 & 58,16 & 2,68 & 38,27 & 2,00 & 28,57 \\
\hline
\end{tabular}

Fuente: elaboración propia.

Intervención sobre el nivel lector de los estudiantes objeto de investigación

La ejecución del primer taller de intervención, de la manera tradicional (el aula organizada en filas), evidenció en los estudiantes el deseo de realizar una actividad distinta a lo que diariamente se vive en el aula, y por disposición del grupo, la realización de los talleres en segunda y tercera sesión se desarrollaron en mesa redonda, lo que posibilitó compartir sus experiencias, opiniones e imaginarios de forma espontánea entre iguales, generándose en el estudiante sentimientos de empoderamiento, al percibirse como elemento importante e integrado en el desarrollo de la actividad pedagógica de lectura crítica. De igual manera, para el docente esta manera de llevar a cabo el proceso de enseñanzaaprendizaje, le permitió orientar de forma directa y cercana al estudiante, la actividad a realizar, al tiempo que se aclaraban dudas y se posibilitó el reconocer de forma inmediata, el grado de entendimiento por parte del estudiante de las temáticas de densidad y flotabilidad. Mediante esta forma de proceder, el estudiante se convierte en el centro del proceso lector (Freire, 1989).
Siguiendo el pensamiento de Freire (1989), esta puesta en práctica, permite al estudiante y al docente, descubrirse, a través de una modalidad de acción cultural, dialógica, problematizadora de sí mismos en su confrontamiento con el mundo social, cultural, histórico y lleno de fenómenos. Esto significa, descubrirse en un sentido profundo, con una percepción distinta del simple desciframiento de signos, donde cada objeto de conocimiento mundo, hombre, cultura, trabajo, planta, animal, van asumiendo un significado que antes no tenían. Reconociéndose entonces como seres transformadores de la realidad, a través de ese trabajo creador. Descubriéndose como hombres que no pueden continuar siendo «objetos» poseídos, y se alcanza así la toma de conciencia de su posición en la sociedad y el mundo.

\section{Evolución del nivel lector en los estudiantes del grupo de investigación}

De la evolución alcanzada por el grupo de investigación, resultados en tablas 6 y 7 , se evidencia un aumento en el nivel de comprensión literal del texto en $8,7 \%$; en el nivel de comprensión inferencial, del 1,5\%; y en el nivel crítico, 
del $1 \%$. De aquí, se hace importante indicar en qué subcategorías de lectura crítica los estudiantes avanzaron considerablemente; en el caso de Valorar la Procedencia del Texto (VPT), se pasó en el pretest de 13,8 \% al postest en 15,6\%, incrementándose en 1,8\% el número de estudiantes que acertaron la pregunta referida a esta subcategoría; para el caso de Asume una Postura Crítica (APC), se pasó de 27,6 \% a $34,4 \%$, incrementándose en $6,8 \%$ el número de estudiantes que acertaron la respuesta, esta subcategoría, es una de las que más interesa, ya que el desempeño en ella es un reflejo del grado de profundidad en lectura crítica que se ha alcanzado a nivel grupal, en palabras de Girón, Jiménez \& Lizcano (2007), el lector es quien tiene

la capacidad de evaluar lo que se ha leído e integrar esa comprensión al conocimiento previo que se tiene del mundo, llegar a conclusiones basándose en razones, ser diligente en la búsqueda de información relevante, razonable en la selección de criterios, para así decidir qué se puede aceptar como verdadero y útil...puede adoptar un punto de vista propio frente al texto. (p. 114).

Tabla 6. Promedio grupal de aciertos y porcentajes por nivel de lectura, pretest-grupo de investigación.

\begin{tabular}{|c|c|c|c|c|c|c|}
\hline & \multicolumn{5}{|c|}{ Aciertos por nivel de lectura, porcentajes y promedios } \\
& Literal & $\begin{array}{l}\text { Porcentaje } \\
\%\end{array}$ & Inferencial & $\begin{array}{c}\text { Porcentaje } \\
\%\end{array}$ & Crítica & $\begin{array}{c}\text { Porcentaje } \\
\%\end{array}$ \\
\hline Promedio grupal & 3,8 & 54,2 & 2,5 & 36,0 & 1,9 & 27,1 \\
\hline
\end{tabular}

Fuente: elaboración propia.

Tabla 7. Resultados promedio de aciertos y porcentajes por nivel de lectura, grupo de investigación - Postest.

\begin{tabular}{|c|c|c|c|c|c|c||}
\hline & \multicolumn{5}{|c|}{ Aciertos por nivel de lectura, porcentajes y promedios } \\
& Literal & $\begin{array}{l}\text { Porcentaje } \\
\%\end{array}$ & Inferencial & $\begin{array}{c}\text { Porcentaje } \\
\%\end{array}$ & Crítica & $\begin{array}{c}\text { Porcentaje } \\
\%\end{array}$ \\
\hline Promedio grupal & 4,4 & 62,9 & 2,6 & 37,5 & 2,0 & 28,1 \\
\hline
\end{tabular}

Fuente: elaboración propia. 
En lo referido a Establecer Relaciones Intertextuales (ERI), se pasó de 37,9 $\%$ a $50 \%$ aumentando el número de estudiantes que acertaron a la respuesta en $12,1 \%$; y en la subcategoría Reconoce Analogías (RA), se pasó de $17,2 \%$ a $37,5 \%$, incrementándose el número de aciertos en 20,3 \%.

Hubo tres subcategorías en las cuales el número de aciertos disminuyó: para el caso de Considerar la Posición del Autor (CPA), se pasó de $20,7 \%$ de aciertos a $9,4 \%$, se disminuyó en 3 el número de aciertos; en la pregunta referida a Contextualizar el Texto (CT), se pasó de $51,7 \%$ a $43,8 \%$, disminuyendo en 1 el número de aciertos; y en Diferenciar entre Hechos y Opiniones (DHO), se pasó de 20,7 \% a 6,3 \% disminuyéndose en 4 el número de aciertos, esta última subcategoría es la que menos número de aciertos registra en el Postest.

Para el caso del nivel de lectura crítica, la tendencia de la subcategoría DHO, está relacionada con la dificultad que presentan los estudiantes para diferenciar entre un hecho y una opinión, situación que fue notoriamente constante y evidenciada en el desarrollo de los talleres de intervención, sin embargo, es de destacar que los resultados presentados obedecen al trabajo individual realizado durante la prueba SABER (pretest y postest) por cada estudiante, como consecuencia de los talleres de intervención ejecutados de forma grupal, por lo que, consideradas las subcategorías del nivel de lectura crítica, desde una mirada global, se evidencia un avance importante y considerable, ya que hubo un incremento en el número de aciertos para 4 de las 7 subcategorías, lo que representa el 57,1 $\%$ de las subcategorías abordadas, y que, por ende, los talleres de intervención trabajados en un ambiente dialógico y mediante el desarrollo colaborativo e interactivo en el aula, ofrece una alternativa en el fortalecimiento de la lectura crítica de los estudiantes.

\section{Conclusiones}

El abordaje de las ciencias naturales y su enseñanza-aprendizaje desde los talleres enfocados en lectura crítica, son una alternativa en el proceso de construcción de conocimientos y conceptualización de las ciencias exactas, debido a que la lectura crítica de la fenomenología científica, implica preguntar, indagar, contrastar, y relacionar los nuevos conceptos con las realidades del contexto del estudiante, hasta conocer de manera certera y profunda, las causas de las situaciones observadas o los problemas planteados, alcanzando de esta manera un mayor grado de aprendizaje significativo y mayor comprensión del medio natural que lo rodea.

La ejecución de talleres enfocados en lectura crítica, direccionados desde el constructivismo y la pedagogía crítica, pueden ser un camino hacia la construcción de habilidades o competencias de lectura crítica en los estudiantes; para el caso particular de esta investigación; los estudiantes vistos de manera grupal, alcanzaron un aumento del 8,7 \% en el nivel de lectura literal, del 1,5\% en el nivel inferencial
El abordaje de las ciencias naturales y su enseñanzaaprendizaje desde los talleres enfocados en lectura crítica, son una alternativa en el proceso de construcción de conocimientos y conceptualización de las ciencias exactas, debido a que la lectura crítica de la fenomenología científica 
y del $1 \%$ en el nivel crítico, avance que es considerable y representativo, ya que tan solo unas décimas en el puntaje obtenido en las pruebas SABER, es decisivo a la hora de ser evaluado tanto a nivel personal; como institucional, en el caso del ISCE.

El desarrollo de talleres de lectura enfocados a la criticidad, fomenta en el estudiante la capacidad de evaluar el texto y, a través de él, al autor; al tiempo que se evalúa a sí mismo, al deber contextualizar el conocimiento y la información que adquiere con su medio natural y sociocultural, logrando extrapolar los conceptos a su realidad, encontrando en ella una aplicación tangible, y una explicación a los sucesos y fenómenos que se dan a su alrededor. Esto fortalece la identidad medio ambiental y socio-cultural al encontrarle sentido al medio donde vive, construyendo así un aprendizaje significativo.

Dentro de la formación docente, es indispensable; que los currículos universitarios cuenten con una asignatura donde se promueva la enseñanza-aprendizaje de la comprensión lectora, su fomento en cuanto a alcanzar niveles profundos de comprensión como el de la lectura crítica, los maestros suelen considerar muy importante el desarrollo de esta habilidad, pero dentro de su formación académica no cuentan o son muy generalizadas las prácticas lectoras que pueden interiorizar y, a su vez, enseñar en el aula.

La actividad lectora, teniendo como eje central el estudiante, sus observaciones y proposiciones en torno a la ejecución del ejercicio, que redunden en espacios agradables para el diálogo, el debate y la expresión de ideas, así como en la participación activa; terminan siendo factores de motivación para que el estudiante se concentre en la práctica lectora, la disfrute y genere el deseo de abordar nuevas actividades de lectura.

La lectura crítica puede ser un camino para que el estudiante empiece a liberar su conciencia, que de alguna manera ha sido alienada y enmarcada en las ideologías transmitidas intencionadamente $\mathrm{o}$ no a través de los textos escritos, los medios de comunicación o los discursos, por lo tanto, la lectura crítica debe constituirse en una práctica educativa transversal, clave no solo para la comprensión, aprehensión y construcción del conocimiento, sino también para la formación de un ser humano crítico del contexto sociopolítico en el que se encuentra y con la posibilidad de desarrollar habilidades para hacer propuestas dirigidas a la transformación de ese entorno y la construcción de una sociedad más humana. 


\section{Referencias}

Aguilar, R. (2011). Propuesta Didáctica para la Enseñanza y Aprendizaje de los Conceptos de Densidad y Presión Abordados en la Educación Básica Secundaria. (Tesis de Maestría). Universidad Nacional de Colombia, Bogotá, Colombia. Recuperado el 19 de octubre de 2017, de http://www.bdigital.unal.edu. co/4884/1/favioyecidaguilarrodriguez.2011.pdf

Alderson, J. C. (2000). Assessing reading. Cambridge: Cambridge University Press.

Alvarado, M. (2007). José Martí y Paulo Freire: aproximaciones para una lectura de la pedagogía crítica. Revista Electrónica de Investigación Educativa, 9(1). Recuperado el 10 de octubre de 2016, de http://redie.uabc.mx/redie/article/ view/157/271

Araujo, J., Betancourt, J., Gómez, J., González, F., \& Pareja, M. (2015). La Pedagogía Crítica el Verdadero Camino Hacia la Transformación Social. (Tesis de Maestría). Universidad de Manizales, Manizales, Colombia. Recuperado el 07 de octubre 2016, de http://ridum.umanizales.edu.co:8080/xmlui/bitstream/ handle/6789/2230/Gonzalez_Francisco_Javier_2015.pdf?sequence=1.

Arias, M., Beltrán, D., \& Solano, M. (2012). La Secuencia Didáctica como Estrategia para Desarrollar Niveles de Lectura Crítica en Estudiantes de Grado Quinto. (Tesis de Especialización). Corporación Universitaria Minuto de Dios(UNIMINUTO), Bogotá, Colombia. Recuperado el 29 de abril de 2016, de http://repository. uniminuto.edu:8080/xmlui/bitstream/handle/10656/2406/TELEC_ AriasIbarraMarthaErminia_2012.pdf?sequence $=2$.

Barral, F. M (1990). ¿Cómo flotan los cuerpos que flotan? Concepciones de los estudiantes. Departamento de Didáctica das Ciencias Experimentales. Santiago de Compostela. Enseñanza de las ciencias, 8(3), 244-250. Recuperado el 19 de octubre de 2017, de http://www.raco.cat/index.php/Ensenanza/ article/download/51333/93081

Cassany, D., Luna M., \& Sanz, G. (1998). Enseñar Lengua. Barcelona: Grao.

Cassany, D. (2003). Aproximaciones a la lectura crítica: teoría, ejemplos y reflexiones. Tarbiya, (32), 113-129. Recuperado el 16 de febrero de 2016, de https://repositori.upf.edu/bitstream/handle/10230/21224/Cassany_ TARBIYA_32.pdf?sequence $=1$ \&isAllowed $=\mathrm{y}$

Chaves, A. (2001). Implicaciones Educativas de la Teoría Sociocultural de Vigotsky. Revista Educación, 25(2), 59-65. Recuperado el 24 de febrero de 2016, de http:// www.uv.mx/personal/yvelasco/files/2012/08/Implicaciones_edcucativas_ de_la_teoria_sociocultural_de_Vigotsky.pdf

Comisión Europea. Dirección general de Educación y Cultura. (2004). Hacia un enfoque de la educación en competencias. Un marco de referencia europeo. 
Recuperado el 10 de febrero de 2011, de http://www.educastur.princast.es/ info/calidad/indicadores/doc/comision_europea.pdf

Elliott, J. (1993). El cambio educativo desde la investigación-acción. Madrid: Morata.

Escuela Normal Superior Antonia Santos. (2009). Informe Ejecutivo del PEI. Sendero de Cambio II.

Freire, P. (1989). La educación como práctica de la libertad. Madrid: Siglo XXI editores.

Freire, P., \& Macedo, D. (1989). Alfabetización. Lectura de la palabra y lectura de la realidad (S. Horvath. Trad.). Barcelona: Paidós-Ministerio de Educación y Ciencia.

García, A. (1998). Historia, epistemología y enseñanza de las ciencias; caso mecánica de fluidos. Enseñanza de las ciencias. Revista de Investigación y Experiencias didácticas, 1255-1259. Recuperado el 20 de octubre de 2017, dehttp://www.raco.cat/index.php/Ensenanza/article/view/293942/382468

Girón, S., Jiménez C., \& Lizcano, C. (2007). ¿Cómo hacer lectura crítica? Colección Cuadernillos. Serie de Gramática. Universidad Sergio Arboleda. Bogotá, Colombia.

Madrigal, A., \& Slisko, J. (2010). Un frasco flota en el agua y se hunde en el aceite: ¿cómo los alumnos de bachillerato explican tales hechos y qué predicen para una situación más compleja? Latin American. Journal of Physics. Education, 4(2), 408-414. Recuperado el 20 de octubre de 2017, de https://dialnet. unirioja.es/descarga/articulo/3696431.pdf

Martínez, R., \& Zea, E. (2004). Estrategias de Enseñanza Basadas en un Enfoque Constructivista. Revista Ciencias de la Educación, 2(24), 69-90. Recuperado el 24 de febrero de 2016, de http://servicio.bc.uc.edu.ve/educacion/revista/ a4n24/4-24-4.pdf

Mazzitelli, C., Maturano, C., Núñez, G., \& Pereira, R. (2006). Identificación de dificultades conceptuales de alumnos y docentes de EGB sobre la flotación de cuerpos. Revista Eureka, 3(1), 33-50. Recuperado el 20 de octubre de 2017, de http://rodin.uca.es/xmlui/bitstream/handle/10498/16223/Mazzitelli_ et_al_2005.pdf?sequence $=1$

Ministerio de Educación Nacional (MEN). (2009). Decreto1290. Bogotá, Colombia. Abril 16 de 2009. Recuperado el 19 de abril de 2017, de http://www. mineducacion.gov.co/1621/articles-187765_archivo_pdf_decreto_1290.pdf

Moll, L. (1993). Vygotsky y la educación (2a Ed.). Buenos Aires: Aique.

Morín, E., Ciurana, E., \& Motta, R. (2006). Educar en la era planetaria. Barcelona, España: Gedisa.

Murillo, F. (2010). Investigación Acción. México: Universidad Autónoma de México. 
Recuperado el 11 de marzo de 2017, de https://www.uam.es/personal_pdi/ stmaria/jmurillo/InvestigacionEE/Presentaciones/Curso_10/Inv_accion_ trabajo.pdf

Serrano de Moreno, M. S. (2008). El Desarrollo de la Comprensión Crítica en los Estudiantes Universitarios: Hacia una Propuesta Didáctica. EDUCERE, 12(42), 505-514. Recuperado el 25 de abril de 2017, de http://www.redalyc. org/pdf/356/35614569011.pdf

Zubiría, J. (2015, 27 de octubre). ¿Democracia sin lectura crítica? Semana.com. [En línea]. Recuperado el 26 de febrero de 2016, de http://www.semana.com/ educacion/articulo/lectura-critica-para-la-democracia/447801-3 